\title{
Discussion: Studying Small Bodies by Means of Molecular Dynamics and Molecular Statistical Theory
}

DOI: $10.1134 / \mathrm{S} 0036024415030127$

The numerous studies on the physicochemical characteristics of solids have long been interpreted conventionally from the viewpoint of classic equilibrium thermodynamics, so there can be little doubt as to the correctness of similar interpretations of the measured or modelled characteristics of small bodies. The diffusion mixing of components in a body (or the relaxation of structural transformations, which also change its local density) naturally accelerates sharply with a reduction in its size. Interpreting the characteristics of small bodies in terms of classic thermodynamics automatically implies an equilibrium state exists inside the bodies of interest and they are in equilibrium with the surrounding medium (thermostat).

However, estimates recently obtained for the characteristic relaxation times of the density of solid elements in spherical specimens with radii from $1 \mathrm{~cm}$ to $1 \mathrm{~nm}$ show that even the simplest vacancy-mediated density equalization mechanism cannot be realized for any real time of an experiment (up to several years). The process is completely inhibited at temperatures $T \sim(0.5-0.7) T_{\mathrm{m}}$ for radii of $10^{-2}-10^{-1} \mathrm{~cm}$ and $T \sim(0.3-0.5) T_{\mathrm{m}}$ for radii of $10^{-7}-10^{-6} \mathrm{~cm}$, where $T_{\mathrm{m}}$ is the melting temperature of a component at $1 \mathrm{~atm}$. Or, there is always a range of temperatures below which it is impossible to experimentally reach an equilibrium state even for very small particles.

Interpreting experimental dependences as functions of size and temperature therefore plays an important role, both thermodynamic and thermophysical, not only for small bodies, but also for macroscopic specimens.

The board of editors invites all concerned specialists engaged in experimental studies and modeling in different fields (solid materials, alloys, vitrification, polymers, biological objects, etc.) to take part in our discussion of the reliability of current thermodynamic data (apart from the ways of measuring them) and how they differ from thermophysical data (depending on the measuring technique).

The articles we publish will present the viewpoints of specialists in molecular dynamics on different characteristics of small solids and compare them to the positions of classic thermodynamics and molecular statistical theory.

The Editorial Board

Translated by E. Glushachenkova 